\title{
Gender based disruptive selection maintains body size polymorphism in Drosophila melanogaster
}

\author{
Jaya Handa, KT Chandrashekara ${ }^{\dagger}$, Khushboo Kashyap, Geetanjali Sageena \\ and MallikarJun N ShaKarad* \\ Department of Zoology, University of Delhi, Delhi 110 007, India \\ ${ }^{\dagger}$ Present address: Institute of Excellence, University of Mysore, Manasagangotri, Mysore \\ 570 006, India
}

*Corresponding author (Email, beelab.ms@gmail.com)

\begin{abstract}
Darwinian fitness in holometabolous insects like the fruit fly Drosophila melanogaster is reported to be positively correlated with body size. If large individuals in a population have higher fitness, then one would expect directional selection to operate leading to uniformly large individuals. However, size polymorphism persists in nature and needs further probing. We assessed the effect of body size on some of the fitness and fitness-related traits in replicate populations of genotypically large, genotypically small and phenotypically small D. melanogaster flies. In this study, the time taken to attain reproductive maturity and copulation duration were independent of fly size. Fecundity and longevity of large females were significantly higher when they partnered genotypically small males than when they were with genotypically larger or phenotypically small males. The increased female longevity when in association with genotypically small males was not due to selective early death of males that would release the female partner from presumed cost of persistent courtship. On the contrary, the genotypically as well as phenotypically small males had significantly higher longevity than large males. The virility of the genotypically small males was not significantly different from that of genotypically large males. Our results clearly show that selection on body size operates in the opposite direction (disruptive selection) for the two genders, thus explaining the persistence of size polymorphisms in the holometabolous insect, Drosophila melanogaster.
\end{abstract}

[Handa J, Chandrashekara KT, Kashyap K, Sageena G and Shakarad MN 2014 Gender based disruptive selection maintains body size polymorphism in Drosophila melanogaster. J. Biosci. 39 609-620] DOI 10.1007/s12038-014-9452-x

\section{Introduction}

In all sexually reproducing organisms, the time taken to attain reproductive maturity (RM) and copulation duration (CD) - the length of time for which a mating pair remains coupled - are important fitness related traits. The copulation process facilitates the transfer of sperms and other male secretary proteins that are required for successful fertilization and production of viable offspring. Body size is one of the most obvious phenotypic trait (Roff 1981) that is shown to be a reliable predictor of fitness and fitness-related traits in many Drosophila species (Lefranc and Bundgaard 2000; Pavkovic-Lucic and Kekic 2013). Body size per se is fixed at the time of emergence in all post-mitotic organisms (Partridge et al. 1987). Further, it is suggested that adult life-history traits are primarily influenced by the energy reserves that are believed to be static throughout the adult life (Roff 1992), perhaps a belief strengthened due to its

Keywords. Body size; disruptive selection; Drosophila; fitness

Abbreviations used: Acps, Accessory gland proteins; AG, Accessory gland; CD, Copulation duration; GL, Genotypically large flies; GS, Genotypically small flies; PS, Phenotypically small flies; RM, Reproductive maturity; SFPs, Seminal fluid proteins; SLC, Standard laboratory conditions; SM, Standard banana-jaggery media 
positive relation with body size (Zwaan et al. 1995; Nunney 1996; Chippindale et al. 1997a; Prasad et al. 2000, 2001). In a study involving 42 Drosophila species, RM in males was shown to be positively correlated with body size and sperm length, while in females RM was not correlated with body size (Pitnick et al. 1995). In Drosophila melanogaster, a key model organism used in understanding many adaptive processes, large males attracted and acquired significantly more mates compared to smaller males due to production of louder and better quality courtship song (Partridge and Farquhar 1983; Partridge et al. 1987). In addition, large males had significantly shorter CD compared to small males (Partridge et al. 1987; Pitnick 1991; Pitnick and Garcia-Gonzalez 2002), perhaps due to presence of higher energy reserves and large reproductive organs that would facilitate transferring required amount of sperms and other reproductive products in a short duration. However, Kraaijeveld et al. (2008) reported $\mathrm{CD}$ to be independent of testes size but inversely related to accessory gland (AG) size. Taken together, the above studies suggest $\mathrm{CD}$ to be a male selected trait (Pitnick 1991; Pitnick and Garcia-Gonzalez 2002). However, it has been reported that the males of many organisms transfer other seminal fluid proteins (SFPs), some of which are known to be detrimental to the fitness of the female partner thus triggering an inter-sexual conflict (Parker 1979; Chapman et al. 2003; Tregenza et al. 2006). Females that were courted and mated by large males had shorter life-span compared to those courted and mated by small males (Partridge et al. 1987; Pitnick 1991; Pitnick and GarciaGonzalez 2002; Chapman et al. 2003; Tregenza et al. 2006). Thus, remaining coupled for a shorter duration with a larger male is advantageous to the female as well, and hence, CD might be a female determined process (Lefranc and Bundgaard 2000). There is considerable ambiguity with respect to the proximate mechanisms that determine time to $\mathrm{RM}$ and who among the mating partners determines $\mathrm{CD}$.

Besides RM and CD, fecundity, longevity and remating frequency (virility) are other fitness and fitness-related traits of great significance for organisms that are engaged in progeny production throughout the life. Fecundity is a shared trait between the female and male partners, while longevity and virility are an individual's traits that are influenced by the reproductive status. The longevity of the reproducing flies is reported to be significantly reduced compared to their virgin siblings (Partridge and Farquhar 1983; Rush et al. 2007; Barnes et al. 2008). Several studies have reported that larger flies have higher fitness compared to their smaller siblings both among males (Partridge et al. 1987; Pitnick 1991; Pitnick and Garcia-Gonzalez 2002) and females (Partridge et al. 1987; Pitnick 1991; Lefranc and Bundgaard 2000; Pitnick and Garcia-Gonzalez 2002). However, male remating frequency, one of the male fitness related traits, is reported to be positively correlated with AG size but uncorrelated with testes and body size (Bangham et al. 2002; Baker et al. 2003).

In short, a large majority of the literature dealing with body size advocates 'bigger is better' hypothesis. According to neo-Darwinian theory of evolution, any trait that has selective advantage should go to fixation over long evolutionary time scale. Hence, if 'bigger is better' for both genders, then one would expect directional selection to operate in favour of large body size and thus result in populations with uniformly large individuals with no or little variability. However, populations of $D$. melanogaster continue to exhibit size polymorphism. In this study, we revisit the 'bigger is better' hypothesis and discuss the possible biological reasons for the persistence of size polymorphism in D. melanogaster.

\section{Materials and methods}

\subsection{Fly lines and their maintenance}

A total of nine D. melanogaster populations were used in this study. Three of the nine were genotypically large $\left(\mathrm{GL}_{1-3}\right)$, three were genotypically small $\left(\mathrm{GS}_{1-3}\right)$ and three were phenotypically small $\left(\mathrm{PS}_{1-3}\right)$. Populations bearing identical numerical subscripts are more closely related to each other than to other populations with which they share a selection regime or maintenance protocol $\left(\mathrm{GL}_{\mathrm{i}}\right.$ and $\mathrm{GS}_{\mathrm{i}}$ are more closely related than $\mathrm{GL}_{\mathrm{i}}$ and $\mathrm{GL}_{\mathrm{j}}$ or $\mathrm{GS}_{\mathrm{i}}$ and $\mathrm{GS}_{\mathrm{j}}$; i,j 1-3). Consequently, populations with identical subscripts were treated as blocks in the statistical analyses (Prasad et al. 2001). The pre-adult as well as the adult stages of all the nine populations used in this study were reared on standard banana-jaggery media (SM) at standard laboratory conditions (SLC) of $25 \pm 1^{\circ} \mathrm{C}$ temperature, 70 $\pm 5 \%$ RH and 24:0 L:D (Chandrashekara and Shakarad 2011), in Powers Scientific Inc. USA, environmental chambers.

The GL populations were on a 3-week egg-to-egg discrete generation cycle, and were reared at a moderate density of 40-60 eggs per vial with $6 \mathrm{~mL}$ SM. Forty vials were maintained per population and incubated for 12 full days at SLC. At the end of 12 days (form the egg collection day) all adults from the 40 vials were transferred to a single pre-labeled breeding cage and provided with ad libitum SM. Fresh SM was provided every alternate day till day 18 from the egg collection day. On day 18, the SM plate was supplemented with live yeast-acetic acid paste. Eggs for starting the next generation were collected on the 21 st day from the preceding egg collection day.

The $\mathrm{GS}_{1-3}$ populations were derived from the respective $\mathrm{GL}_{1-3}$ populations by selecting for faster per-adult development and reproduction at late age. The GS fly maintenance was identical to GL, excepting that the egg density was 60 80 eggs per $6 \mathrm{~mL}$ SM vial, 160 vials were maintained per 
population and only the first $12-15$ of the emerging flies from each vial were transferred to pre-labeled breeding cages. Flies of each replicate population were maintained in two sister cages in order to avoid adult crowding till $\sim 50 \%$ adult mortality. Eggs were collected from surviving flies of the two sister cages, combined and redistributed into 160 vials so as to avoid independent evolution in each of the sister cages for a given replicate population. The number of breeding adults in both GL and GS was $\sim 1600$ per population. The GS populations had under gone 110 generations of selection before being used in this study and their egg-adult development time was shorter by $\sim 40 \mathrm{~h}$ than GL populations, and were considerably smaller in size (see results). In order to eliminate all non-genetic effects the GL and GS populations were passed through common rearing conditions (Chippindale et al. 1997b) for one generation and egg collection was staggered by the developmental time difference in order to obtain assay flies of comparable chronological age (Prasad et al. 2001).

The $\mathrm{PS}_{1-3}$ populations were generated from the respective $\mathrm{GL}_{1-3}$ populations by dispensing 400 eggs into vials containing $3 \mathrm{~mL} \mathrm{SM}$ over-laid on $3 \mathrm{~mL}$ non-nutritive agar. The total volume was regulated at $6 \mathrm{~mL}$ so as to keep the moisture conditions in the vial constant across different fly populations. The high egg density coupled with low food resulted in emergence of phenotypically small flies. The emerging flies were sorted according to their gender and maintained as virgins in holding vials containing $\sim 4 \mathrm{~mL}$ SM before being used in further assays. The $\mathrm{PS}_{1-3}$ populations had the same genotype as that of the corresponding GL populations.

\subsection{Fly size and lipid content}

The size of the flies was estimated by weighing of flies, as many studies have reported a good correspondence between weight and other size measures. On emergence, the assay flies were sorted based on gender into groups of 10 individuals and transferred to pre-labeled clean empty dry vials, dried at $70^{\circ} \mathrm{C}$ for $36 \mathrm{~h}$ and weighed to obtain dry weight. The dry flies were transferred to corresponding pre-labeled 1.5 $\mathrm{mL}$ Eppendroff micro-centrifuge tubes containing $1.2 \mathrm{~mL}$ diethyl ether (Merck, GR grade) and put on a gel rocker, set to $2000 \mathrm{rpm}$ to extract ether soluble lipids. The lipids were extracted over $36-40 \mathrm{~h}$ with two ether changes at $12 \mathrm{~h}$ intervals. After the last ether change the flies were washed in excess of ether, dried for $2 \mathrm{~h}$ at room temperature and weighed to obtain lipid free weight of flies. The dry weight and lipid free dry weight were used to obtain the lipid content in the flies. Five replicate vials per sex per replicate population were set up. In all there were 90 replicate vials with 900 flies.

\subsection{Reproductive maturity}

The time lag between the 'time of emergence' and 'initiation of copulation' is considered as 'reproductive maturity (RM)'. Freshly emerged adults were paired with the selftype 3- to 5-day-old adults (assumed to be reproductively mature and ready to copulate) of the opposite sex. Thirty pairs were set up per gender, per replicate population.

\subsection{Copulation duration}

The average time lag between the coupling and decoupling of the mating pairs is expressed as copulation duration (CD). In order to test whether the $\mathrm{CD}$ is governed by either the female or the male, mating pairs were set up by using 3- to 5day-old virgin flies from the holding vials. In this experiment, a self type (female $\times$ male: GL $\times$ GL, PS $\times \mathrm{PS}, \mathrm{GS} \times$ GS) or cross type (female $\times$ male: GL $\times$ PS, GL $\times$ GS, PS $\times$ GL, GS $\times$ GL) pairing was done. In addition, a pair of virgin GL females was provided to single GL or GS male for 5 rounds and $\mathrm{CD}$ was recorded in every round. Twenty such triplet ( 2 females +1 male) pairings per replicate population were set up.

\subsection{Male virility assay}

Male virility was tested as the average number of males that could mate with at least five females. In this experiment, 3to 5-day-old virgin males from the GL and GS populations were used. The females were from the corresponding GL populations, as the purpose of this experiment was to test the expression of the full potential of the male in a common female background. The females of the GL populations are the largest among the available stocks in our laboratory and are thus believed to aid in full expression of reproduction related traits. Initially, 30 virgin males were separated in to 2 $\mathrm{mL}$ SM mating vials, provided with two virgin females each and were given $2 \mathrm{~h}$ time duration for courtship and copulation. On observation of copulation between a pair, the nonparticipating female was carefully aspirated out of the mating vial. All copulating males were transferred to fresh mating vials after they had decoupled and provided with two fresh virgin females at the start of the next $2 \mathrm{~h}$ cycle. This process was repeated 5 times. The experiment was terminated after the fifth round as we exhausted the stock of virgin females. Sirot et al. (2009) reported that the male exhausts its accessory gland products in 3 matings and hence all matings post third to be blank. In order to ascertain effective copulations, all copulating females were retained in the original mating vial after decoupling and allowed to oviposit for $24 \mathrm{~h}$. The females were discarded at the end of 
$24 \mathrm{~h}$ and vials were incubated at SLC for a week, and observed for pupation.

\subsection{Fecundity and longevity assay}

Some of the male accessory gland proteins (Acps) are reported to influence the female longevity in addition to fecundity (Chapman et al. 1995; Wolfner 2002). In order to ascertain the effect of male size on the female longevity and fecundity, GL females were paired with either GL, GS or PS males. For fecundity assay, 20 single pairs were set per replicate population. Flies were transferred without anesthesia to fresh SM vials every $24 \mathrm{~h}$, and the eggs laid during the previous $24 \mathrm{~h}$ were counted using binocular microscope and recorded. The daily egg counts were carried out till the death of the female fly. While, for longevity assay, 20 vials of 4 pairs ( 4 females +4 males) were set up per replicate population. Census was carried out on a daily basis. The dead flies were aspirated out, gender identified and recorded. The surviving flies were transferred to fresh SM vials every alternate day. The census was continued till the death of the last fly.

\subsection{Testes and accessory gland size measurement}

Three- to five-day-old virgin males from the holding vials were transferred to fresh clean pre-labeled empty vials, freeze killed and stored for $6 \mathrm{~h}$ at $-20^{\circ} \mathrm{C}$. The flies were dissected in chilled $1 \mathrm{X}$ PBS solution. The entire male reproductive system was extracted and spread in 1X PBS. Images were captured using $5 \mathrm{X}$ stereoscope camera and saved in TIFF format. The area of accessory gland (AG) and testes was measured using ImageJ (Schneider et al. 2012) program. Twenty flies per replicate population were dissected.

\subsection{Ovary size measurement}

3-5 day old females were kept in $-20^{\circ} \mathrm{C}$ for 5-6 days before dissection. Flies were dissected in chilled 1X PBS solution. The entire female reproductive system was extracted and ovaries were spread in 1X PBS. Images were captured using $5 \mathrm{X}$ stereoscope camera and saved in TIFF format. The area of the ovary was measured using ImageJ (Schneider et al. 2012) program. Twenty flies per replicate population were dissected.

\subsection{Statistical analyses}

Data from all the assays were subjected to separate mixedmodel analyses of variance (ANOVA), treating block as a random factor and population type as a fixed factor crossed with block. In all cases, the population means were used as the units of analysis and, therefore, only fixed-factor effects and interactions can be tested for significance (Prasad et al. 2001). The difference among treatment means was compared using Tukey-Kramer Minimum Significant Difference (MSD) Test (Sokal and Rolf 1995). The difference between adult survival curves was analyzed using Kaplan-Meier logrank test (Fisher and Van 1993). Graphical presentations of all results except survival probability values are indicated as 'mean \pm s.e.'

\section{Results}

\subsection{Dry weight and lipid content}

Dry weight of both, the females $\left(\mathrm{F}_{2,6}=138.364, p<0.000\right)$ and the males $\left(\mathrm{F}_{2,6}=175.03, p<0.000\right)$ were significantly different among the three types of fly populations. A post-hoc analysis using Tukey-Kramer MSD test indicated that females from GL populations were significantly heavier $(\mathrm{MSD}=53.143, p<0.01)$ than GS and PS, that were not significantly different amongst themselves (figure 1A). Among the male flies GL were significantly heavier ( $\mathrm{MSD}=32.861, p<0.01$ ), than GS as well as PS, while PS and GS were significantly different at 5\% level of significance $(\mathrm{MSD}=2.521)$ (figure $1 \mathrm{~B})$. Lipid content among the GL and PS females were not significantly different amongst themselves, but were significantly higher than GS females $(\mathrm{MSD}=23.404, p<0.01)$ (figure $1 \mathrm{C})$. The males of GS had significantly lower (MSD=11.792, $p<0.01)$ lipid levels compared to that of GL and PS males, that were significantly different among themselves at 5\% level of significance $(\mathrm{MSD}=8.082)$ (figure 1D).

\subsection{Reproductive maturity}

There was no significant effect of fly type (GL/GS/PS) $\left(\mathrm{F}_{2,4}=1.001, p=0.444\right)$ and fly type $\times$ gender interaction $\left(\mathrm{F}_{2,4}=3.356, p=0.139\right)$ on RM. However, there was a significant effect of fly gender on RM $\left(\mathrm{F}_{1,2}=2377.534, p=0.000\right)$. Irrespective of the fly type, on an average the females took 18.33 hours, while males took $19.9 \mathrm{~h}$ to become reproductively active.

\subsection{Copulation duration}

There was no significant effect of fly size $\left(\mathrm{F}_{2,6}=0.850\right.$, $p=0.47)$, gender $\left(\mathrm{F}_{1,3}=9.17, p=0.056\right)$ and gender $\times$ size interaction $\left(\mathrm{F}_{2,6}=0.42, p=0.67\right)$ on $\mathrm{CD}$. In an independent assay where GL and GS males were provided sequentially with up to 10 virgin GL females (5 successive $2 \mathrm{~h}$ intervals with 2 females at each interval), the CD was not significantly 
(A)

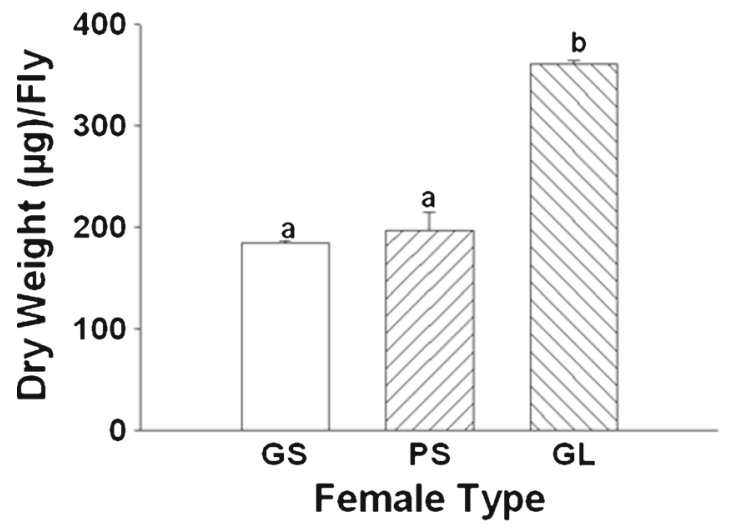

(C)

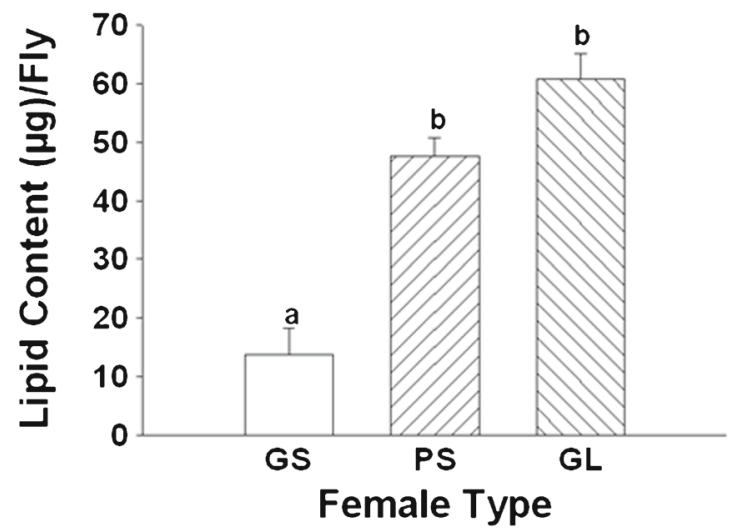

(B)

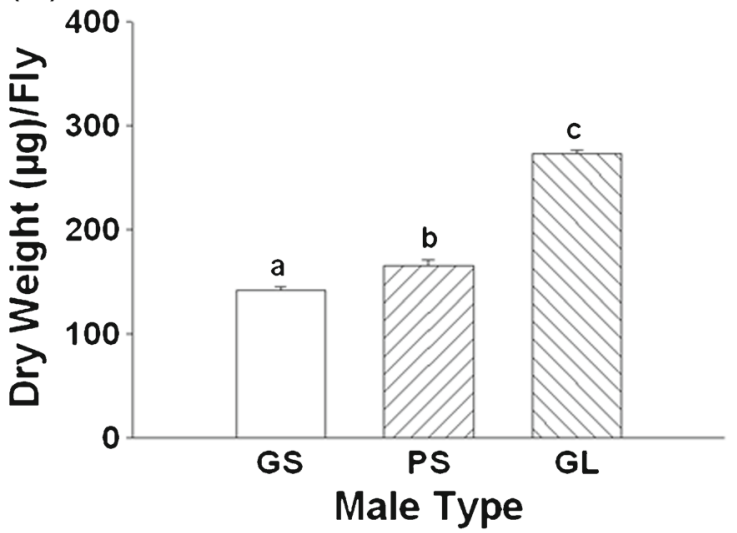

(D)

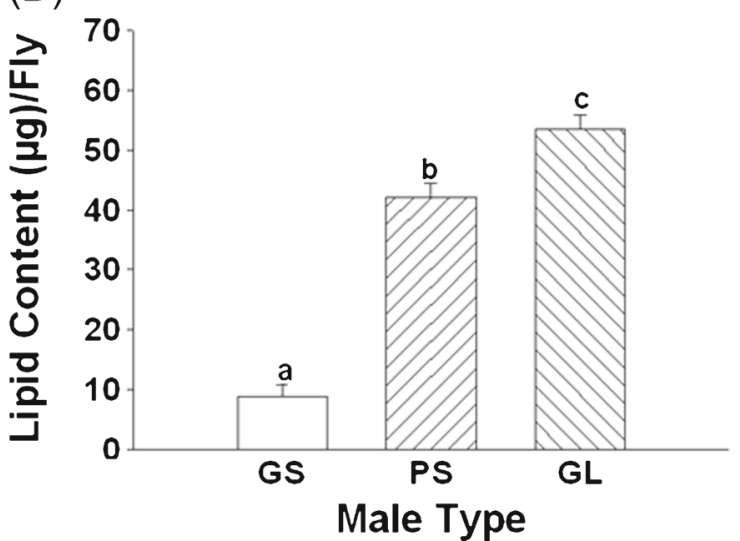

Figure 1. Average ( \pm s.e.) dry weight and lipid content per fly, GL: Genotypically/ Phenotypically Large, PS: Phenotypically Small and GS: Genotypically Small. Bars with same letters are not statistically significantly different while those with different letters are statistically significantly different. (A) Average dry weight of different type of female flies, (B) average dry weight of different type of male flies, (C) average lipid content of different type of female flies, (D) average lipid content of different type of male flies.

different between the two male types $\left(\mathrm{F}_{1,2}=2.29, p=0.27\right)$. The sequence of the $2 \mathrm{~h}$ assay interval also did not have significant effect on average $\mathrm{CD}\left(\mathrm{F}_{4,8}=1.413, p=0.313\right)$.

\subsection{Male virility and mating latency}

Male virility was tested as the average number of males that could mate with at least five females. There was no significant difference in the virility of GL and GS males $\left(\mathrm{F}_{1,2}=4.945, p=0.156\right)$. In addition, there was no significant difference in the proportion of effective copulations measured as the number of copulations leading to production of viable eggs among the GL and GS male partnered females $\left(\mathrm{F}_{4,4}=1.697, p=0.31\right)$. Although there was overall significant effect of the male type on the mating latency $\left(\mathrm{F}_{1,2}=68.21\right.$, $p=0.014$ ), there was no significant difference in the slopes of the regression curves of time lag between successive matings $\left(t_{(2), 6}=1.899, p>0.10\right)$.

\subsection{Testes and accessory gland size}

There was a significant effect of fly type on both the testes $\left(\mathrm{F}_{2,5}=33.631, p<0.001\right)$ and accessory gland $\left(\mathrm{F}_{2,5}=20.532\right.$, $p<0.003)$ size. The testes of GL males were significantly larger $(\mathrm{MSD}=8797.91, p<0.01)$ than both GS and PS males, that did not differ significantly amongst themselves (figure 2A). Similarly, the AG size of GL were significantly larger than $\mathrm{GS}(\mathrm{MSD}=8655.546, p<0.05)$ and $\mathrm{PS}$ $(\mathrm{MSD}=12147.33, p<0.01)$, that did not differ amongst themselves (figure $2 \mathrm{~B}$ ) 

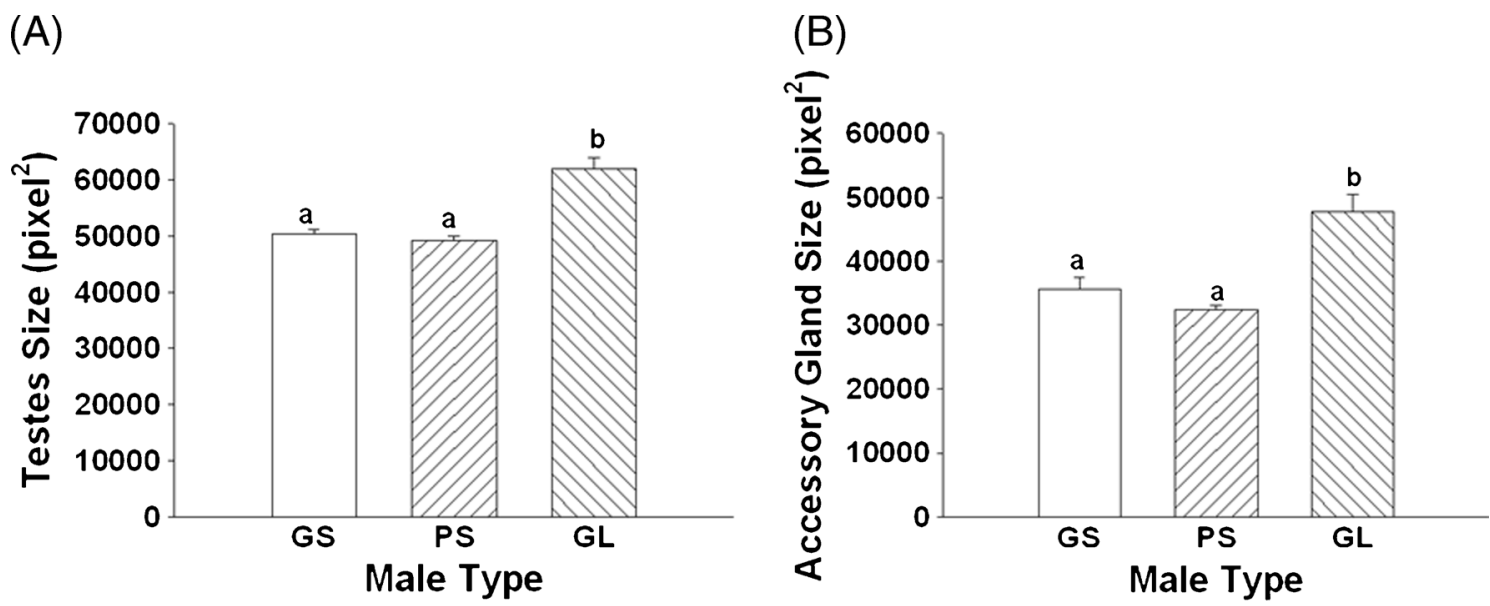

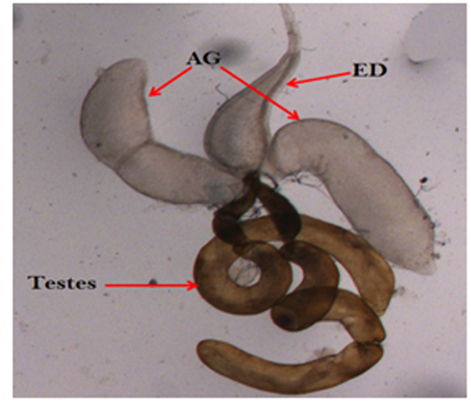

GS

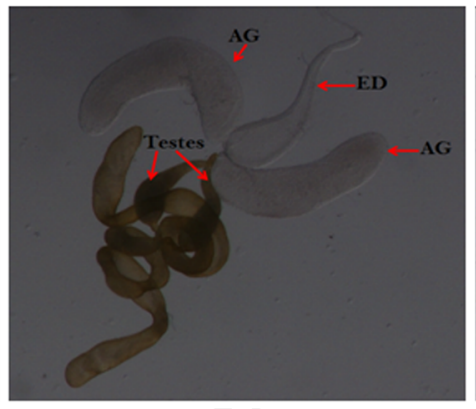

PS

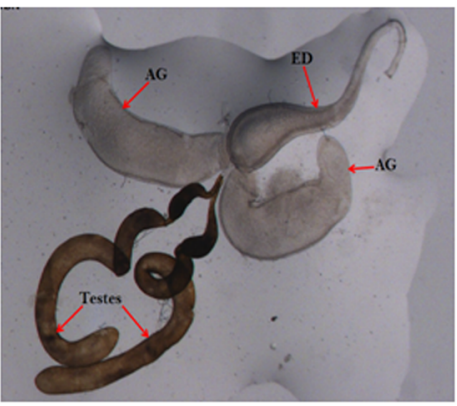

GL

Figure 2. Average ( \pm s.e. $)$ male gland sizes. Bars with same letters are not statistically significantly different while those with different letters are statistically significantly different. (A) Testes size, (B) accessory gland size. Inset: testes, accessory gland (AG) and ejaculatory duct (ED) images of different types of males. There were no visible anatomical differences in the reproductive organs of the three male types.

\subsection{Ovary size}

There was a significant effect of fly type on the average (of both left and right) ovary area $\left(\mathrm{F}_{2,4}=53.965, p<0.001\right)$. The ovaries of GL females were the largest (136177.7 pixel squared) followed by GS (82698.84 pixel squared) and PS (37960.82 pixel squared) females (Figure 3). The TukeyKramer MSD values are 33736.73 and 54353.63 for $5 \%$ and $1 \%$ level of significance respectively.

\subsection{Fecundity and longevity}

The type of male partner significantly influenced the average total lifetime fecundity of the female $\left(\mathrm{F}_{2,6}=33.305, p<0.005\right)$. GL females produced significantly higher number of eggs $\left(\mathrm{MSD}_{\alpha 0.05}=93.775 ; \mathrm{MSD}_{\alpha 0.01}=136.826\right)$ when paired with GS (722.81) than GL (501.65) or PS (512.31) males (figure 4A). When the male type was held constant (in this case GL males) and fecundity of the different female types was assessed, there was a significant effect of female type on fecundity $\left(\mathrm{F}_{2,6}=64.340, p<0.000\right)$. The total fecundity of GL (501.65) and GS (533.33) were not significantly different $\left(\mathrm{MSD}_{\alpha 0.05}=72.43\right)$, but were significantly higher than PS females (287.21, $\mathrm{MSD}_{\alpha 0.01}=105$. 682) (figure 4B).

The type of male partner significantly affected the longevity of the reproducing female. GL females lived significantly longer when paired with GS males $\left(\chi^{2}=14.317\right.$, $p<0.001$ ) than with $\mathrm{GL}$ males (figure $5 \mathrm{~A}$ ). There was no significant difference in the longevity of GL females $\left(\chi^{2}=0.903, p>0.5\right)$ when they were paired with GL or PS males (figure 5B). GS ( $\chi^{2}=7.061, p<0.01$; figure 5C) and PS $\left(\chi^{2}=9.344, p<0.005\right.$; figure 5D) males lived significantly longer than GL males.

\section{Discussion}

Attainment of reproductive maturity (RM) marks the beginning of reproduction and hence is an important life-history 


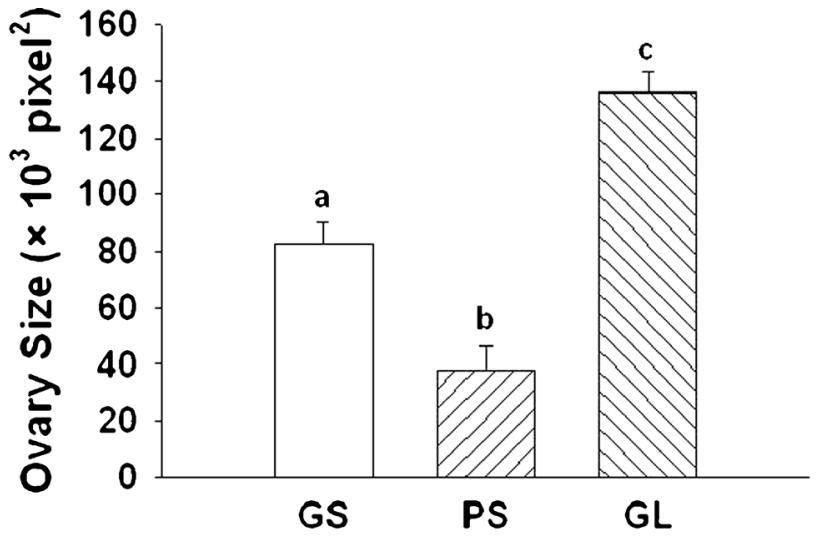

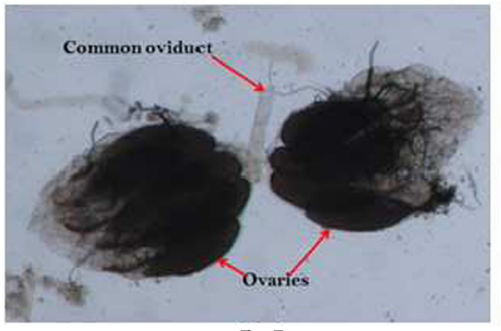

GS

Female Type

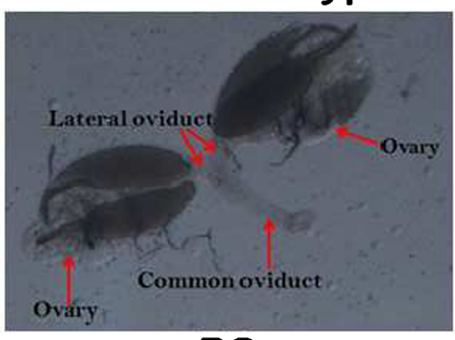

PS

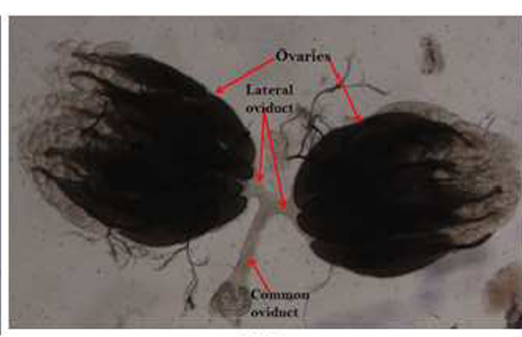

GL

Figure 3. Average ( \pm s.e.) ovary size. Bars with same letters are not statistically significantly different while those with different letters are statistically significantly different. Inset: ovary images of different types of females. There were no visual differences in the ovary of GL and GS females, while the ovary of PS were visibly deformed.

trait. There is no clarity on the proximate mechanisms determining time to RM. A study with stalk-eyed fly, Cyrtodiopsis dalmanni showed RM in males to be negatively correlated with AG size (Baker et al. 2003). Another study involving 42 species of Drosophila showed RM to be positively correlated with testis size and sperm length (Pitnick et al. 1995) and testis mass shows a positive relationship with body mass (Pitnick 1996), while body mass is reported to be correlated with body size (Prasad et al. 2000; Prasad and Joshi 2003). Body size is a complex, quantitative phenotypic trait (Blanckenhorn 2000) that is suggested to be the most comprehensive predictor of fitness, especially in Drosophila fruit flies (for a complete list, see table 1 of Pavkovic-Lucic and Kekic 2013). However, in this study, RM was not effected by AG, testes, ovary or the over all body size, suggesting that RM perhaps is a species and/or
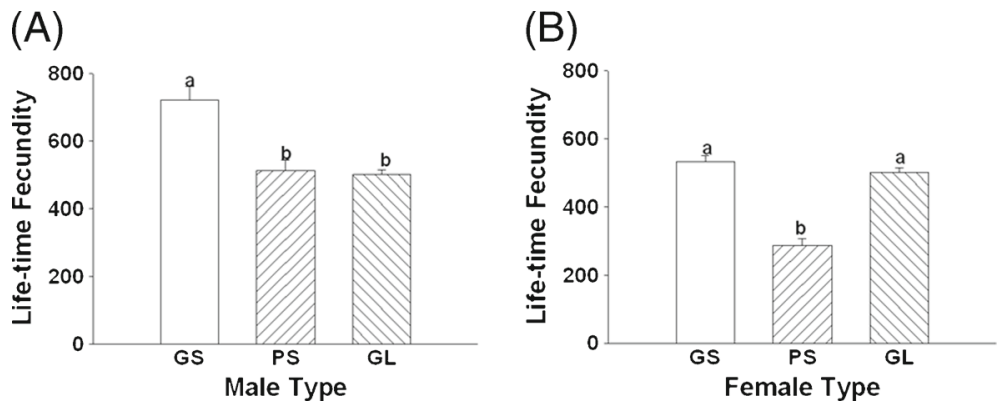

Figure 4. Average life-time fecundity. Bars with same letters are not statistically significantly different while those with different letters are statistically significantly different. (A) Fecundity of GL female when in association with different type of males. (B) Fecundity of GL male when in association with different type of females. 
(A)

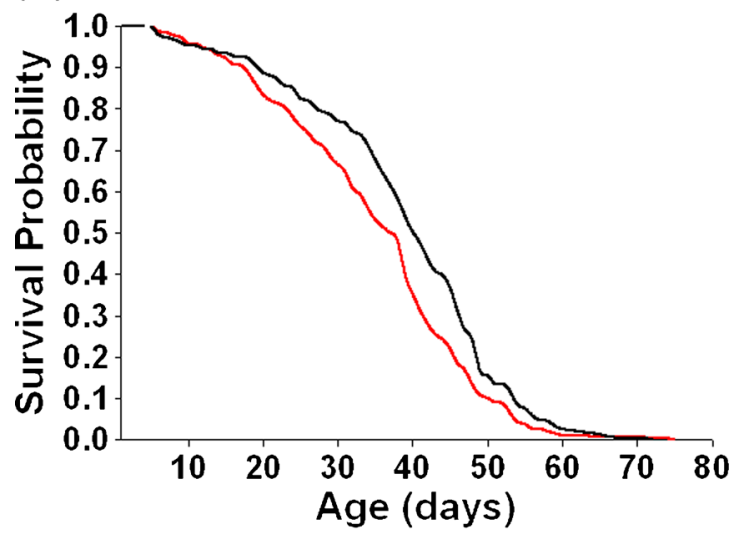

(C)

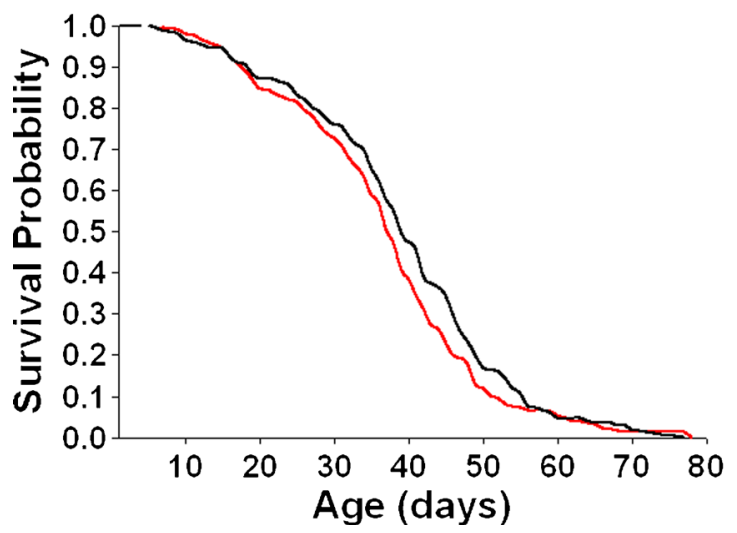

(B)

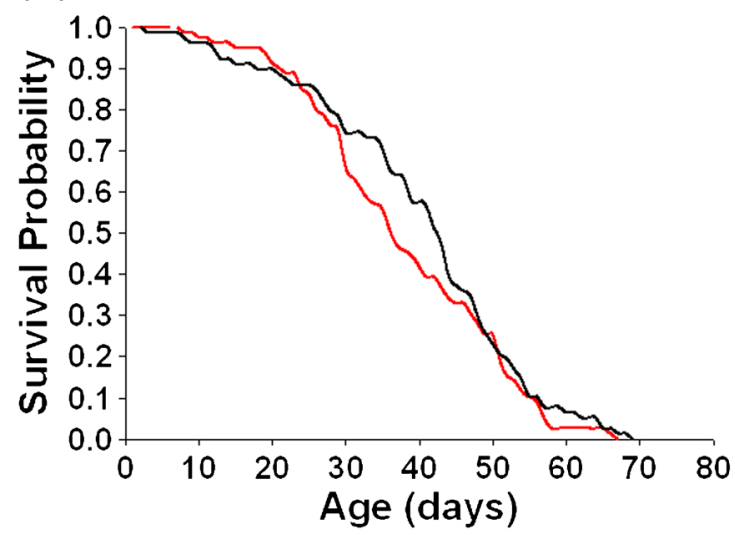

(D)

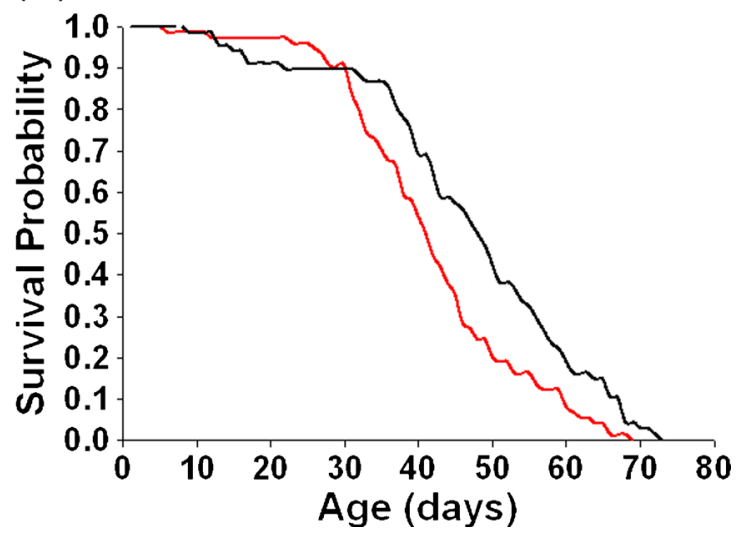

Figure 5. Kaplan-Meier survival probability curves for GL females when paired with GL (red) or GS (black) males (A), and GL (red) or PS (black) males (B). Kaplan-Meier survival probability curves for GL (red) and GS (black) males (C), and GL (red) or PS (black) males (D).

strain specific trait that has been optimized over the course of evolutionary time. Further, trait correlations can greatly differ within and across species, and it is the within species correlations and tradeoffs that can facilitate or constrain evolution (Prasad and Joshi 2003).

$\mathrm{CD}$ is another trait that has direct and strong effect on the fitness of both the male as well as the female partners. Primarily, $\mathrm{CD}$ has been viewed as an index of the male ejaculate investment (Friberg 2006; Bretman et al. 2013) and hence might be under the control of the male partner with larger males having shorter CD compared to smaller males (Macbean and Parsons 1967; Pitnick 1991; Pitnick and Garcia-Gonzalez 2002). However, other studies reported $\mathrm{CD}$ to be positively correlated with female body size (Lefranc and Bundgaard 2000) but uncorrelated with male body size (Lefranc and Bundgaard 2000; Imroze and Prasad 2011). In the present study, there was no significant effect of either the female or the male body size as well as AG or testes size on $\mathrm{CD}$. The differences in our results and those of others could be due to (i) the differing genetic architecture of the fly populations that have perhaps taken different evolutionary trajectories (Archer et al. 2003; Chippindale et al. 2003; Phelan et al. 2003; Prasad and Shakarad 2004), (ii) differences in fly rearing and maintenance conditions, (iii) differences in selection and assay environments (Ackermann et al. 2001), and/or (iv) differences in the sample sizes. The previous studies have used a single population with a sample size of 30 individuals, while our study has used a total of 9 populations (3 replicate populations for each of the 3 types) with a sample size of at least 30 for each population, and hence our results are more robust than others. Besides, in $D$. melanogaster, although the transfer of sperms is completed with in the first 6-8 $\mathrm{min}$, the act of remaining coupled lasts for about $20 \mathrm{~min}$ (Gilchrist and Partridge 2000). The variation in $\mathrm{CD}$ is suggested to indicate a variation in the amount of Acps transferred during mating, and that small males are expected to transfer less quantity of Acps compared to larger males (Imroze and Prasad 2011) owing to their small 
(A)

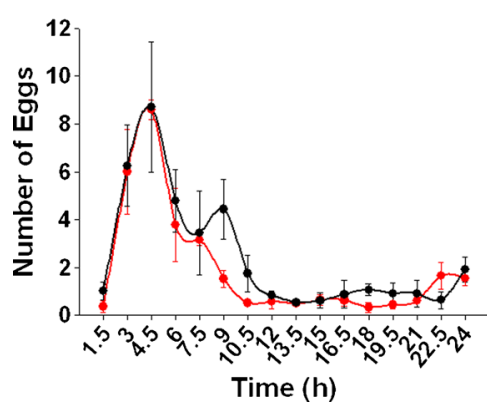

(B)

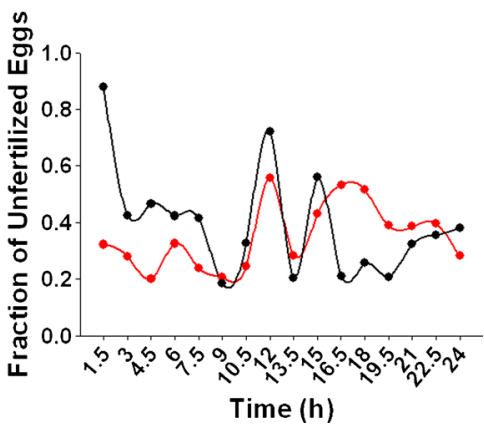

Figure 6. Average number of eggs laid at every $1.5 \mathrm{~h}$ interval by GL females when with GL (red) and GS (black) males during the first $24 \mathrm{~h}$ post mating (A). Fraction of unfertilized eggs laid by females during the first $24 \mathrm{~h}$ post mating with GL (red) and GS (black) males (B).

reproductive organ sizes (figure 2). However, our results showed that $\mathrm{CD}$ is not affected by any of the reproductive organ size or the over all body size, suggesting that CD might be another important life-history-related trait that is species and/or strain specific.

Acps are primarily shown to alter the female physiology. Some of the visible changes in the mated females are: (i) reduced motivation to mate with other males, (ii) increased fecundity and (iii) reduced female life-span (Chapman et al. 1995; Wolfner 2002). A number of studies have reported that the females mated to larger males have reduced fecundity (Pitnick 1991; Pitnick and Garcia-Gonzalez 2002; Friberg 2006; Imroze and Prasad 2011). In the present study the GL females paired with GS males had significantly higher fecundity (figure 4) and longevity (figure 5) compared to either those that were paired with GL (large) or PS (phenotypically small) males. It has been reported that simple exposure of females to non-mating males significantly reduces the female survival (Partridge and Fowler 1990), perhaps due to costs imposed by courtship harassment. It is possible that the GS males had significantly short life-span owing to their small size and thus released the GL females

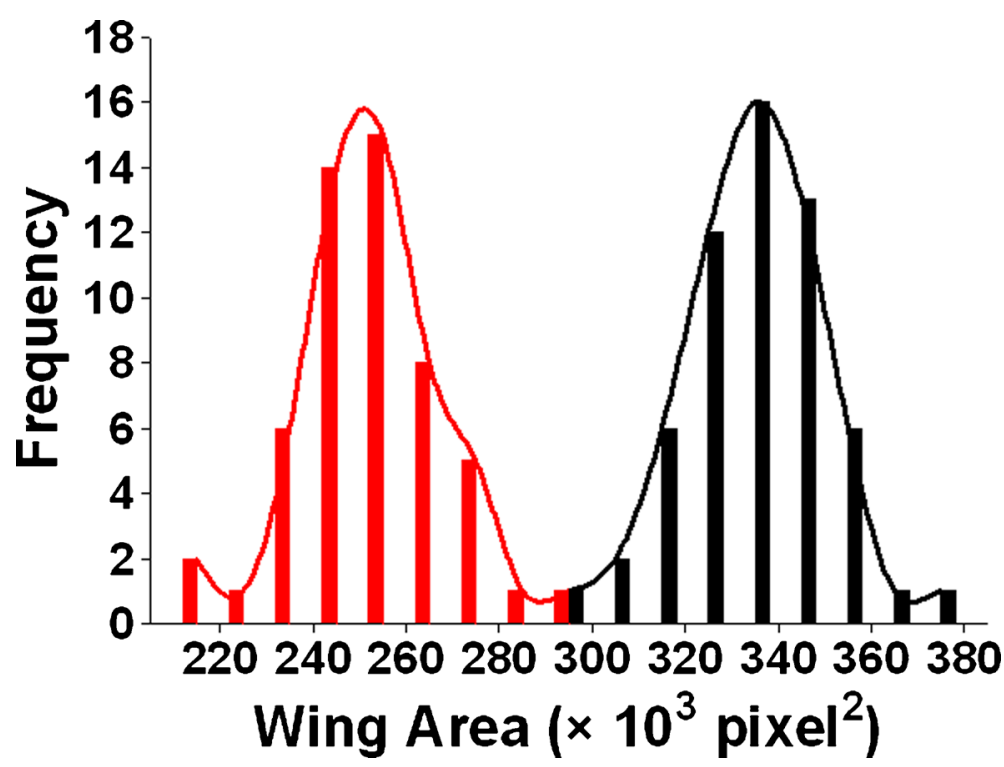

Figure 7. Frequency distribution of average (of left and right) wing area of male (red) and (red) female (black) GL flies. The combined plot of the two genders shows a distinctly bi-modal distribution, with the distributions for the two genders being significantly different $(t=28.594, p<0.001)$ from each other. 
from courtship costs. However the GS males had significantly higher survival probability compared to GL males (figure 5C), suggesting that a mechanism other than simple release from courtship cost is responsible for the higher survival probability of the GL females. The nonsignificantly different fecundity and longevity of the females partnered with GL and PS males suggest that the quantity of the sperm or other SFPs do not greatly influence these traits in the female but perhaps the quality of SFPs do, through altered male physiology (Imroze and Prasad 2011). A 24 $\mathrm{h}$ time series analysis of the average number of eggs laid by females when partnered with GS and GL males showed a difference in the oviposition profile between 6 and $12 \mathrm{~h}$ post copulation with a peak at $9 \mathrm{~h}$ (figure $6 \mathrm{~A}$ ) - a time duration by which packaging of sperms and Acp70A is completed (Scott 1987; Peng, et al. 2005). Further, the large proportion of eggs laid by females partnered with GS males were unfertilized (figure 6B), supporting the hypothesis that ovulin (Acp26Aa) is involved in releasing eggs from the female system immediately post mating (Heifetz et al. 2000). These results clearly suggest that Acp26Aa and Acp70A, the two Acps that are primarily responsible for flushing out eggs from the female system could have evolved to become more efficient in the GS males. Further, GL female partnered with GS males had significantly reduced death rate at every chronological age (figure 5A) suggesting that some of the Acps that are harmful to the female could have evolved towards becoming less toxic. Although our results clearly support Wolfner's (2002) view that Acps are the fastest evolving proteins in Drosophila species, the molecular details of the specific Acps in our populations need to be characterized.

Several studies in the past have reported higher remating frequency for large males compared to their smaller siblings (Partridge and Farquhar 1983; Pitnick 1991), perhaps owing to shorter CD and mating latency (Sisodia and Singh 2004) thus maximizing their overall fitness. However, it has been reported that males exhaust their SFPs in 3 mating and have to remain sexually inactive for 3 consecutive days to attain effective copulation capability (Sirot et al. 2009). In this study, the slopes of the regression lines for mating latency and the number of males that were able to mate with at least five females were not significantly different between the GL and GS populations. The virility of GS males was comparable to that of GL males despite having one-fifth the amount of lipids than the GL males suggesting that there is no fitness advantage to large males. Similar results were reported for $D$. littoralis (Aspi and Hoikkala 1995) and D. pseudoobscura (Markow and Ricker 1992). Interesingly, in D. montana small males had mating advantage over large males (Aspi and Hoikkala 1995). In conclusion, our results clearly establish the fact that RM and CD are species and/or strain specific traits that are unaltered by phenotypic manipulation. Further, our results also show that large females have higher fitness only when in association with genotypically small males, indicating a gender specific disruptive selection for body size (figure 7) that perhaps is responsible for persistence of sexual size polymorphism in D. melanogaster. The very presence of sexual dimorphism is, in and of itself professed to be an evidence that the two sexes have had a history of disruptive selection (Abbott et al. 2010).

\section{Acknowledgements}

We thank two anonymous reviewers for their critical comments that helped in improving the quality of presentation. This research was supported by Council of Scientific and Industrial Research, Government of India EMR grant and University of Delhi R and D grant to MS. KTC and KK thank the Council of Scientific and Industrial Research for Post-doctoral and Junior Research Fellowships respectively. GS thanks University Grants Commission for Junior Research Fellowship. JH thanks Department of Zoology, University of Delhi, for Non-NET Fellowship.

\section{References}

Abbott JK, Bedhomme S and Chippindale AK 2010 Sexual conflict in wing size and shape in Drosophila melanogaster. J. Evol. Biol.. 23 1989-1997

Ackermann M, Bijlsma R, James AC, Partridge L, Zwaan BJ and Stearns SC 2001 Effects of assay conditions in life history experiments with Drosophila melanogaster. J. Evol. Biol. 14 199-209

Archer MA, Phelan JP, Beckman KA and Rose MR 2003 Break down in correlations during laboratory evolution. II. Selection on stress resistance in Drosophila populations. Evolution 57 536-543

Aspi J and Hoikkala A 1995 Male mating success and survival in the field with respect to size and courtship song characters in Drosophila littoralis and D. montana (Diptera: Drosophilidae). J. Insect Behav. 8 67-87

Baker RH, Denniff M, Futerman P, Fowler K, Pomiankowski A and Chapman T 2003 Accessory gland size influences time to sexual maturity and mating frequency in the stalk-eyed fly, Cyrtodiopsis dalmanni. Behav. Ecol. 14 607-611

Bangham J, Chapman T and Partridge L 2002 Effects of body size, accessory gland and testis size on pre and postcopulatory success in Drosophila melanogaster. Anim. Behav. 64 915-921

Barnes AI, Wigby S, Boone JM, Partridge L and Chapman T 2008 Feeding, fecundity and lifespan in female Drosophila melanogaster. Proc. R. Soc. B 275 1675-1683

Blanckenhorn WU 2000 The evolution of body size: what keeps organisms small? The Quart. Rev. Biol. 75 385-407

Bretman A, Westmancoat JD, Gage MJG and Chapman T 2013 Costs and benefits of lifetime exposure to mating rivals in male Drosophila melanogaster. Evolution 67 2413-2422 
Chandrashekara KT and Shakarad MN 2011 Aloe vera or resveratrol supplementation in larval diet delays adult aging in the fruit fly, Drosophila melanogaster. J. Gerontol. 66 965-971

Chapman T, Liddle LF, Kalb JM, Wolfner MF and Partridge L 1995 Cost of mating in Drosophila melanogaster females is mediated by male accessory gland products. Nature 373 241-244

Chapman T, Arnqvist G, Bangham J and Rowe L 2003 Sexual conflict. Trends. Ecol. Evol. 18 41-47

Chippindale AK, Alipaz JA, Chen HW and Rose MR 1997a Experimental evolution of accelerated development in Drosophila. 1. Developmental speed and larval survival. Evolution 51 15361551

Chippindale AK, Leroi AM, Saing H, Borash DJ and Rose MR 1997b Phenotypic plasticity and selection in Drosophila life history evolution. 2. Diet, mates and the cost of reproduction. J. Evol. Biol. 10 269-293

Chippindale AK, Ngo AL and Rose MR 2003 The devil in the details of life-history evolution: instability and reversal of genetic correlations during selection on Drosophila development. J. Genet. 82 133-145

Fisher LD and Van BG 1993 Biostatistics: A methodology for the health sciences (New York: Wiley) pp 786-843

Friberg U 2006 Male perception of female mating status: its effect on copulation duration, sperm defense and female fitness. Anim. Behav. 72 1259-1268

Gilchrist AS and Partridge L 2000 Why it is difficult to model sperm displacement in Drosophila melanogaster: the relation between sperm transfer and copulation duration. Evolution 54 534-542

Heifetz Y, Lung O, Frongillo EA and Wolfner MF 2000 The Drosophila seminal fluid protein Acp26Aa stimulates release of oocytes by the ovary. Curr. Biol. 10 99-102

Imroze K and Prasad NG 2011 Mating with large males decreases the immune defense of females in Drosophila melanogaster. J. Genet. 90 427-434

Kraaijeveld K, Denniff M, Baker RH and Chapman T 2008 The evolution of sperm and non-sperm producing organs in male Drosophila. Biol. J. Linn. Soc. 94 505-512

Lefranc A and Bundgaard J 2000 The influence of male and female body size on copulation duration and fecundity in Drosophila melanogaster. Hereditas 132 243-247

Macbean IT and Parsons PA 1967 Directional selection for duration of copulation in Drosophila melanogaster. Genetics $56 \quad 233-239$

Markow TA and Ricker JP 1992 Male size, developmental stability, and mating success in natural populations of three Drosophila species. Heredity 69 122-127

Nunney L 1996 The response to selection for fast larval development in Drosophila melanogaster and its effect on adult weight: an example of a fitness trade-off. Evolution $\mathbf{5 0}$ 1193-1204

Parker GA 1979 Sexual selection and sexual conflict; in Sexual selection and reproductive competition in insects (eds) MS Blum and NA Blum (London: Academic Press) pp 123-166

Partridge L and Farquhar M 1983 Lifetime mating success of male fruitflies (Drosophila melanogaster) is related to their size. Anim. Behav. 31 871-877
Partridge L and Fowler K 1990 Non-mating costs of exposure to males in female Drosophila melanogaster. J. Insect Physiol. 36 419-425

Partridge L, Ewing A and Chandler A 1987 Male size and mating success in Drosophila melanogaster: the roles of male and female behaviour. Anim. Behav. 35 555-562

Pavkovic-Lucic S and Kekic V 2013 Developmental temperature, body size and male mating success in fruit flies, Drosophila melanogaster (Diptera: Drosophilidae). Eur. J. Entomol. 110 31-37

Peng J, Chen S, Busser S, Liu H, Honegger T and Kubli E 2005 Gradual release of sperm bound sex-peptide controls female postmating behavior in Drosophila. Curr. Biol. 15 207-213

Phelan JP, Archer MA, Beckman KA, Chippindale AK, Nusbaum TJ and Rose MR 2003 Breakdown in correlations during laboratory evolution. I. Comparative analyses of Drosophila populations. Evolution 57 527-535

Pitnick S 1991 Male size influences mate fecundity and remating interval in Drosophila melanogaster. Anim. Behav. 41 735-745

Pitnick S 1996 Investment in testes and the cost of making long sperms in Drosophila. Am. Nat. 148 57-80

Pitnick S and Garcia-Gonzalez F 2002 Harm to females increases with male body size in Drosophila melanogaster. Proc. R. Soc. Lond. B 269 1821-1828

Pitnick S, Spicer GS and Markow TA 1995 How long is a giant sperm? Nature 375109

Prasad NG and Joshi A 2003 What have two decades of laboratory life-history evolution studies on Drosophila melanogaster taught us? J. Genet. 82 45-76

Prasad NG and Shakarad MN 2004 Genetic correlations: transient truths of adaptive evolution. J. Genet. 83 3-6

Prasad NG, Shakarad M, Anitha D, Rajamani M and Joshi A 2001 Correlated responses to selection for faster development and early reproduction in Drosophila: The evolution of larval traits. Evolution 55 1363-1372

Prasad NG, Shakarad M, Gohil VM, Sheeba V, Rajamani M and Joshi A 2000 Evolution of reduced pre-adult viability and larval growth rate in laboratory populations of Drosophila melanogaster selected for shorter development time. Genet. Res. 76 249-259

Roff DA 1981 On being the right size. Am. Nat. 118 405-422

Roff DA 1992 The evolution of life histories: theory and analysis (New York: Chapman and Hall)

Rush B, Sandver S, Bruer J, Roche R, Wells M and Giebultowicz J 2007 Mating increases starvation resistance and decreases oxidative stress resistance in Drosophila melanogaster females. Aging Cell 6 723-726

Schneider CA, Rasband WS and Eliceiri KW 2012 NIH Image to ImageJ: 25 years of image analysis. Nat. Methods $9671-$ 675

Scott D 1987 The timing of the sperm effect on female Drosophila melanogaster receptivity. Anim. Behav. 35 142-149

Sirot LK, Buehner NA, Fiumera AC and Wolfner MF 2009 Seminal fluid protein depletion and replenishment in the fruit fly, Drosophila melanogaster: an ELISA-based method for tracking individual ejaculates. Behav. Ecol. Sociobiol. 63 1505-1513

Sisodia S and Singh BN 2004 Size dependent sexual selection in Drosophila ananassae. Genetica 121 207-217 
Sokal RR and Rolf JF 1995 Biometry: the principles and practice of statistics in biological research, 3rd ed (Freeman and Company)

Tregenza T, Wedell N and Chapman T 2006 Introduction. Sexual conflict: a new paradigm? Phil. Trans. R. Soc. B 361 229-234
Wolfner MF 2002 The gifts that keep on giving: physiological functions and evolutionary dynamics of male seminal proteins in Drosophila. Heredity 88 85-93

Zwaan BJ, Bijlsma R and Hoekstra RF 1995 Artificial selection for development time in Drosophila melanogaster in relation to the evolution of aging: direct and correlated responses. Evolution 49 635-648

MS received 13 January 2014; accepted 02 June 2014

Corresponding editor: SuBHASH C LAKHOTIA 